\title{
Análisis de Estabilidad Probabilístico del Talud de la Cantera Espinal - Juliaca
}

\section{Probabilistic Analysis of Slope Stability Espinal Quarry - Juliaca}

\begin{abstract}
Fredy Alonso Valeriano Nina
Facultad de Ingeniería de Minas, Universidad Nacional del Altiplano - Puno - Perú

DOI: https://doi.org/10.33017/RevECIPeru2014.0006/

\section{Resumen}

El presente estudio fue realizado en la cantera Espinal de la ciudad de Juliaca en el departamento de Puno, consiste en determinar los valores de seguridad que rigen la estabilidad o inestabilidad en un talud rocoso; factor de seguridad (FS) o reducción de la resistencia al corte (SRF) y la probabilidad de falla (PF), para lo cual se han utilizado los métodos generales de análisis de estabilidad de taludes en roca; Análisis cinemático y métodos numéricos. Se realizó análisis probabilísticos por el método de estimación de puntos para mayor confiabilidad de los resultados de análisis con métodos numéricos. Teniendo como resultados en el análisis cinemático una probabilidad de plano de deslizamiento por volteo directo de $44.41 \%$, los valores de seguridad promedio dado por los análisis probabilísticos con métodos numéricos en SRF son; para condiciones estáticas FS o SRF 2.27 y PF $0.0 \%$ y para condiciones seudoestáticas FS o SRF 1.21 y PF 2.98\%. Por lo cual concluimos que el talud se encuentra estable en condiciones estáticas y seudoestáticas, tomando en consideración el resultado del análisis cinemático realizado se han observado grietas de tracción en la cresta del talud que fallaran por volteo directo dependiendo a la intensidad de los factores desencadenantes.
\end{abstract}

Descriptores: Macizo rocosos, análisis probabilístico, factor de seguridad (FS), reducción de la resistencia al corte (SRF), probabilidad de falla (PF).

\section{Abstract}

This study was carried out in the Espinal highway of Juliaca city in the state of Puno, which consists in to determinate the safety values that controls the stability or instability of a rocky slope; safety factor (FS) or shear strength reduction (SRF) and failure probability (PF). Due to this, general methods of rock slope stability were used; cinematic analysis and numerical methods. Probabilistic analyses were conducted by the method of points' estimation for more reliability in the results of numerical methods. Having in the cinematic analysis results a probability of glide plane by direct toppling of $44.41 \%$, the values of average safety obtained by the probabilistic analysis with numerical methods in SFR are; for static conditions FS or SRF 2.27 and PF $0.0 \%$ and for pseudostatical conditions a FS or SRF 1.21 and PF 2.98\%. Due to this, we concluded that the slope is stable in static and pseudostatic conditions, considering the results of cinematic analysis, were observed tension cracks on the top of the slope which failed by direct toppling depending of the intensity of trigger factors.

Keywords: Rock Mass, probabilistic analysis, safety factor (FS), stress reduction factor (SRF), failure probability (PF).

\section{Introducción}

Diferentes tipos de rotura en taludes son asociados con diferentes estructuras presentes en el macizo rocoso y estas son más importantes en un estudio de análisis de estabilidad de taludes en roca debido a que estos controlan la estabilidad o inestabilidad de los mismos. 
Además la medición de las propiedades del macizo rocoso, sobre todo in situ, es una tarea muy difícil. Excepto en las superficies expuestas (que están generalmente limitados en comparación con el volumen de diseño que afectan la roca y que puede no ser representativo de un volumen de material geológico), las características de macizo rocoso, tales como las juntas no son directamente observables. Incluso cuando las propiedades se pueden determinar fácilmente, inexactitudes en la medición y las diferencias entre el laboratorio y el comportamiento a escala de campo pueden generar un error considerable. Como resultado, por lo cual diremos que la ingeniería de las excavaciones en roca implica grandes incertidumbres.

La simulación estadística ofrece un medio para hacer frente con la incertidumbre. Se puede cuantificar la incertidumbre y estimar las probabilidades de ocurrencia de diferentes resultados. Por lo tanto, puede ayudar a los ingenieros a desarrollar diseños y soluciones más reales y económicas.

\section{Metodología}

Consideramos a la presente investigación dentro del diseño metodológico experimental, cuantitativa continua.

La presente investigación se realizó en la Cantera Espinal ubicada en el distrito de Juliaca, provincia de San Román, Departamento de Puno. Teniendo como población a toda la cantera espinal, las muestras están relacionadas a aspectos físicos de los macizos rocosos presentes en la cantera.

Las variables independientes más importantes son la geometría del talud, datos de campo (Dirección de buzamiento, buzamiento, altura y pendiente del talud, dirección del talud), datos de laboratorio (Angulo de fricción, cohesión, resistencia a la compresión uniaxial, densidad, módulos elásticos, otros).

Las variables dependientes son; la probabilidad de plano de deslizamiento para los análisis cinemáticos y el FS o SRF para los análisis por métodos numéricos.

La metodología para un análisis probabilístico de estabilidad en taludes se resume en la Fig. 1.

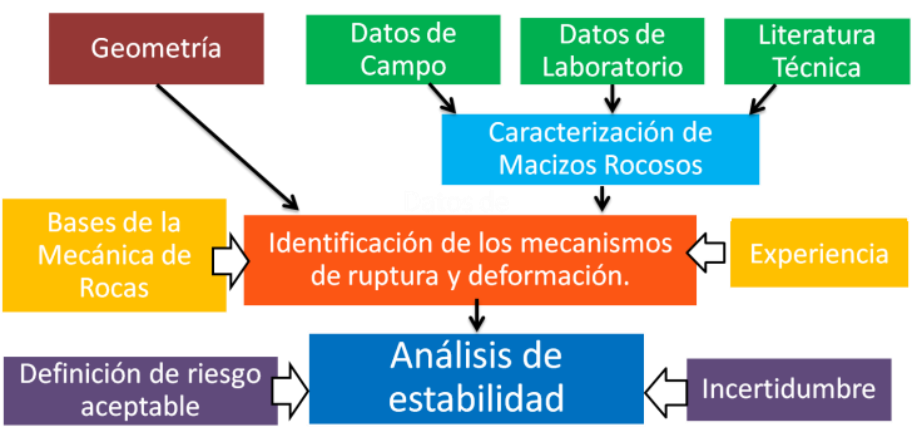

Figura 1: Esquema de la metodología de análisis de estabilidad en taludes en roca, con especial referencia a los conceptos de riesgo e incertidumbre.

Dentro del análisis de estabilidad de taludes los factores geométricos son; la altura del talud y el ángulo del talud. Para lo cual se realizó el levantamiento topográfico de los bordes del talud Espinal con GPSs. Y se modelo en 3D para realizar los cortes del talud en 2D en el software Minesight v.6.0. Versión educativa. (Imagen 1).

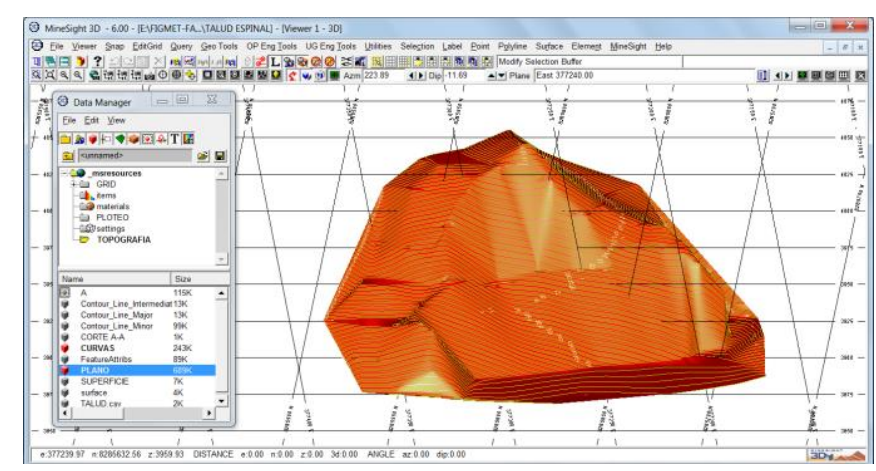

Imagen 1: Modelamiento en 3D de la cantera Espinal - Juliaca.

Dentro de la litología presente en la cantera Espinal tenemos a las fonolitas superior, medio e inferior del grupo Mitú, las areniscas cuarzosas del grupo Ambo y las lutitas del grupo Cabanillas. (Fig. 2). Observándose una falla normal en la parte este del talud.

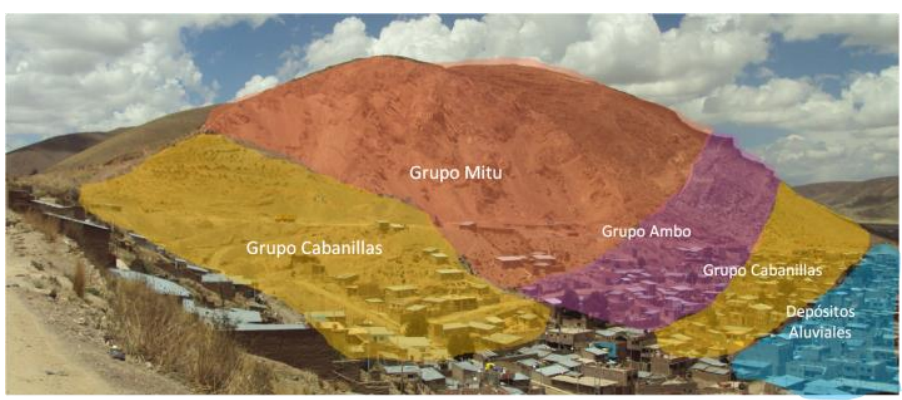

Figura 2: Litología presente en la cantera EspinalJuliaca.

Los datos tomados en el campo fueron las actitudes del macizo rocoso tales como la dirección de 
buzamiento y buzamiento, dirección del pie y la cresta del talud.

Los datos de laboratorio fueron obtenidos en los laboratorios de Mecánica de Rocas y Suelos de la Facultad de Ingeniería de Minas - UNAP. Realizándose 16 ensayos de compresión Uniaxial, 8 ensayos de carga puntual y 6 ensayos de corte directo en discontinuidades. (Tablas 1 y 2 ).

Tabla 1: Resultados de los ensayos de laboratorio para las rocas del grupo Mitu.

\begin{tabular}{|l|c|c|c|}
\hline \multirow{2}{*}{$\begin{array}{c}\text { Resultados de los } \\
\text { ensayos de laboratorio } \\
\text { Grupo Mitu }\end{array}$} & $\begin{array}{c}\text { Propiedades } \\
\text { Indice }\end{array}$ & \multicolumn{2}{|c|}{$\begin{array}{c}\text { Propiedade } \\
\text { s Mecánicas }\end{array}$} \\
\cline { 2 - 5 } & $\mathrm{Y}\left(\mathrm{gr} / \mathrm{cm}^{3}\right)$ & UCS & LPT \\
\hline Numero de resultados & 12 & 8 & 4 \\
\hline Valor máximo, MAX & 2.59 & 91.52 & 77.20 \\
\hline Valor mínimo, MIN & 2.51 & 65.37 & 63.60 \\
\hline Mediana, MED & 2.53 & 70.92 & 68.45 \\
\hline Valor promedio, MEAN & 2.53 & 74.86 & 69.43 \\
\hline $\begin{array}{l}\text { Desviación estándar, } \\
\text { SDEV }\end{array}$ & 0.02 & 10.51 & 6.52 \\
\hline $\begin{array}{l}\text { Coeficiente de variación, } \\
\text { CV }\end{array}$ & 1.21 & 1.20 & 0.43 \\
\hline
\end{tabular}

Tabla 2: Resultados de los ensayos de laboratorio para las rocas del grupo Ambo.

\begin{tabular}{|l|c|c|c|}
\hline \multirow{2}{*}{$\begin{array}{c}\text { Resultados de los } \\
\text { ensayos de laboratorio } \\
\text { Grupo Ambo }\end{array}$} & $\begin{array}{c}\text { Propiedades } \\
\text { Indice }\end{array}$ & \multicolumn{2}{c|}{$\begin{array}{c}\text { Propiedades } \\
\text { Mecánicas }\end{array}$} \\
\cline { 2 - 5 } & $\mathrm{Y}\left(\mathrm{gr} / \mathrm{cm}^{3}\right)$ & UCS & LPT \\
\hline Numero de resultados & 12 & 8 & 4 \\
\hline Valor máximo, MAX & 2.54 & 73.97 & 63.60 \\
\hline Valor mínimo, MIN & 2.48 & 34.41 & 58.60 \\
\hline Mediana, MED & 2.51 & 59.78 & 60.90 \\
\hline Valor promedio, MEAN & 2.52 & 57.37 & 61.00 \\
\hline $\begin{array}{l}\text { Desviación estándar, } \\
\text { SDEV }\end{array}$ & 0.02 & 14.66 & 2.27 \\
\hline $\begin{array}{l}\text { Coeficiente de variación, } \\
\text { CV }\end{array}$ & -0.11 & -0.48 & 0.17 \\
\hline
\end{tabular}

La caracterización se realizó en campo por los métodos RMR y $Q$ de Barton en una hoja Excel y en el laboratorio de Software Minero de la Facultad de Ingeniería de Minas - UNAP. Por el método de caracterización GSI en el programa RocData v. 4.0. de Rocscience.

La identificación de los mecanismos de ruptura fueron observados en el campo, siendo necesario mencionar las grietas de tracción vistas en la cresta del talud Espinal. (Foto 1).

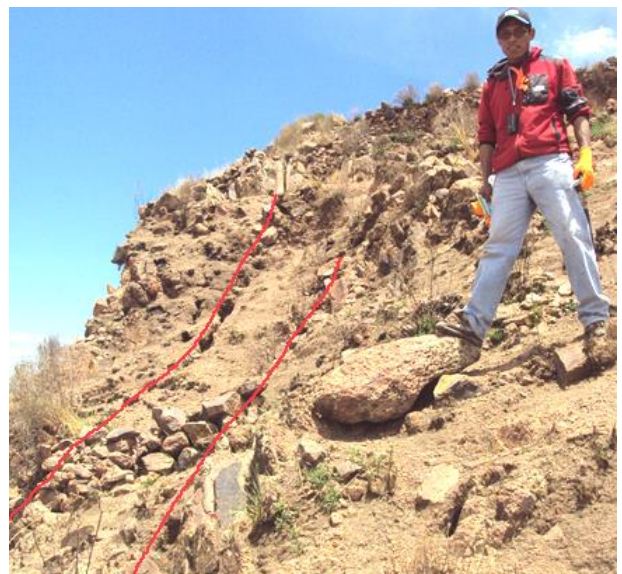

Foto 1: Grietas de tracción en la cresta del talud.

Los análisis de estabilidad de Taludes fueron realizados por los métodos generales para el análisis de estabilidad de taludes en roca; análisis cinemático y análisis numérico.

Todos los análisis de estabilidad fueron realizados en el Gabinete de Software Minero de la Facultad de Ingeniería de Minas - UNAP. En los software de Rocscience con licencia de educación universitaria FIM.

Los análisis cinemáticos se analizaron estadísticamente con los datos obtenidos en campo de las caracterizaciones geomecánicas, en el software Dips v. 6.0 de Rocscience. Por los criterios de ruptura siguientes. Por rotura planar total, rotura planar con restricción, rotura en cuña, rotura por volteo flexural y rotura por volteo directo.

Los análisis por métodos numéricos en 2D se realizó por el método probabilístico de estimación puntual, en condiciones estáticas y seudoestáticas, en el software Phase ${ }^{2}$ v.8.0 de Rocscience por el método de elementos finitos.

Tomando en consideración 0.11 el factor de sismicidad para los análisis seudoestático.

El nivel freático para el análisis probabilístico por métodos numéricos está considerado a un $75 \%$ saturado y $25 \%$ no saturado.

\section{Resultados}

Los resultados obtenidos por la caracterización geomecánica del talud en la etapa de campo, se observan 4 familias de discontinuidades. (Imagen 2). 


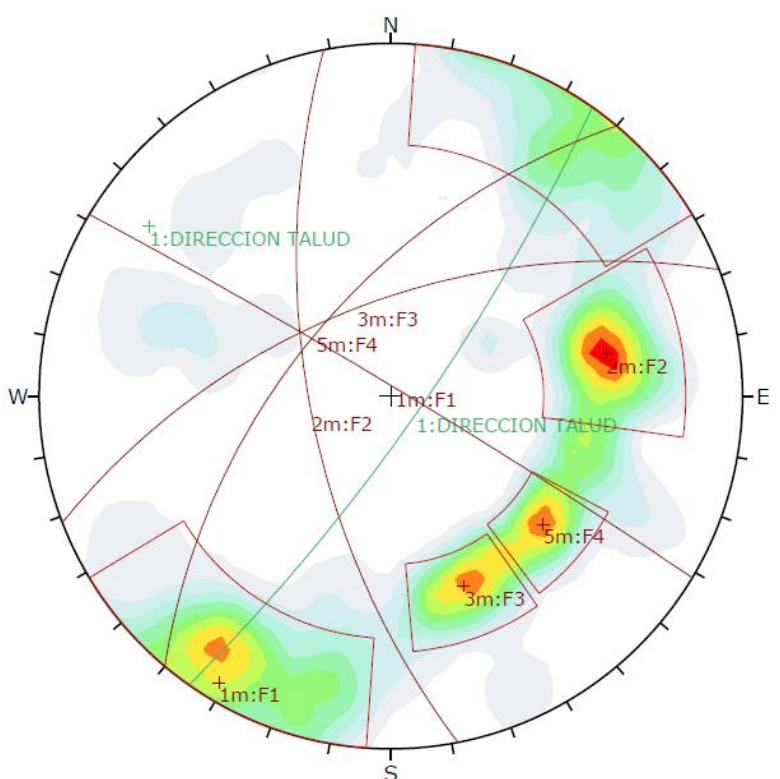

Imagen 2: Densidad de familias, diagrama de polos y dirección del talud.

\subsection{Análisis cinemático}

Para este análisis consideramos los siguientes valores. (Tab. 3).

Tabla 3: Datos para el análisis probabilístico cinemático.

\begin{tabular}{|l|c|}
\hline \multicolumn{2}{|c|}{ DATOS ANÁLISIS CINEMÁTICO } \\
\hline Numero de datos (DipDir/Dip) & 500 \\
\hline $\begin{array}{l}\text { Familia de discontinuidades 1 } \\
\text { (DipDir/Dip) }\end{array}$ & $122 / 87$ \\
\hline $\begin{array}{l}\text { Familia de discontinuidades } 2 \\
\text { (DipDir/Dip) }\end{array}$ & $80 / 26$ \\
\hline $\begin{array}{l}\text { Familia de discontinuidades 3 } \\
\text { (DipDir/Dip) }\end{array}$ & $157 / 31$ \\
\hline $\begin{array}{l}\text { Familia de discontinuidades 4 } \\
\text { (DipDir/Dip) }\end{array}$ & $129 / 32$ \\
\hline Altura del talud & $256 \mathrm{~m}$ \\
\hline Pendiente del talud & $60^{\circ}$ \\
\hline Dirección de Buzamiento del talud & $425^{\circ} \mathrm{E}$ \\
\hline Angulo de fricción & $46^{\circ}$ \\
\hline
\end{tabular}

Teniendo como resultado la probabilidad de plano de deslizamiento (PPD) en porcentajes (Tab. 4), identificando así el modo de ruptura e inestabilidad del talud.

Tabla 4: Resultado de la probabilidad de plano de deslizamiento en porcentajes.

\begin{tabular}{|l|c|}
\hline \multicolumn{2}{|c|}{ RESULTADOS ANÁLISIS CINEMÁTICO } \\
\hline \multicolumn{1}{|c|}{ TIPO DE ROTURA } & PPD (\%) \\
\hline Análisis cinemático por rotura en cuña & $2.63 \%$. \\
\hline $\begin{array}{l}\text { Análisis cinemático de rotura por volteo } \\
\text { flexural }\end{array}$ & $1.60 \%$. \\
\hline
\end{tabular}

\begin{tabular}{|l|c|}
$\begin{array}{l}\text { Análisis cinemático de rotura por volteo } \\
\text { directo }\end{array}$ & $44.41 \%$ \\
\hline $\begin{array}{l}\text { Análisis cinemático de ruptura por volteo } \\
\text { oblicuo }\end{array}$ & $14.51 \%$ \\
\hline $\begin{array}{l}\text { Análisis cinemático de ruptura por volteo } \\
\text { directo en base plana }\end{array}$ & $4.20 \%$. \\
\hline
\end{tabular}

El talud de la cantera Espinal tendrá una mayor tendencia a fallar por una ruptura tipo volteo directo con un $44.41 \%$ de probabilidad de plano de deslizamiento, considerando también el volteo de tipo oblicuo con $14.51 \%$. (Imagen 3 ).

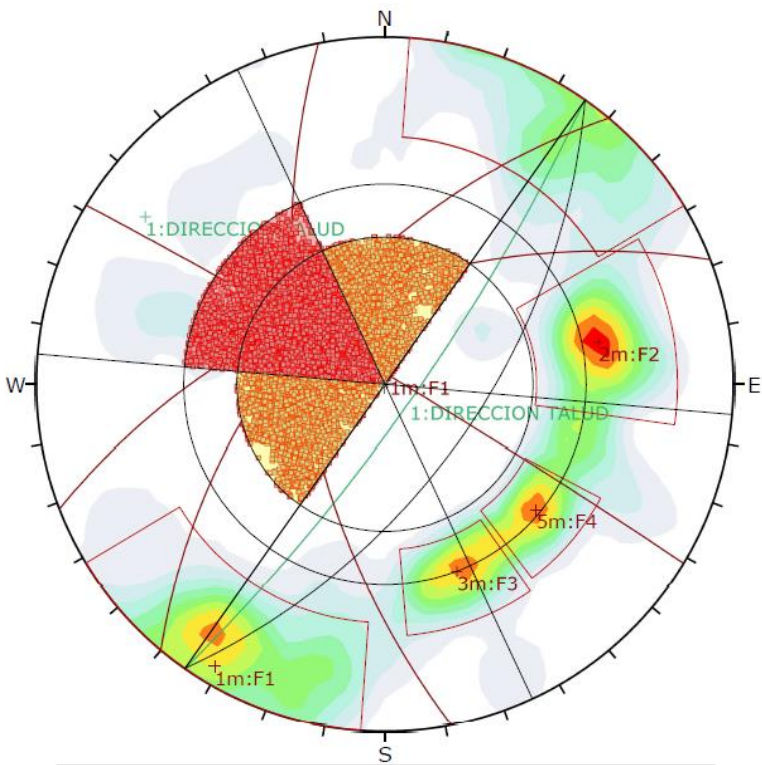

\begin{tabular}{|r|l|c|c|}
\hline Kinematic Analysis & Direct Toppling \\
\hline Slope Dip & 60 \\
\hline Slope Dip Direction & 125 \\
\hline Friction Angle & $46^{\circ}$ \\
\hline Lateral Limits & $30^{\circ}$ & \\
\hline \multicolumn{5}{|c|}{} & Critical & Total & $\%$ \\
\hline Direct Toppling (Intersection) & 55393 & 124729 & $44.41 \%$ \\
\hline Oblique Toppling (Intersection) & 18093 & 124729 & $14.51 \%$ \\
\hline Base Plane (All) & 21 & 500 & $4.20 \%$ \\
\hline
\end{tabular}

Imagen 3: Análisis cinemático de rotura por tipos de volteo directo.

\subsection{Análisis probabilístico por métodos numéricos}

Los análisis se realizaron por el método probabilístico de estimación de puntos con el método de elementos finitos. (Imagen 4).

El SRF en condiciones estáticas fue 2.37, con una probabilidad de falla de $0.0 \%$. Considerando este resultado muy confiable. 


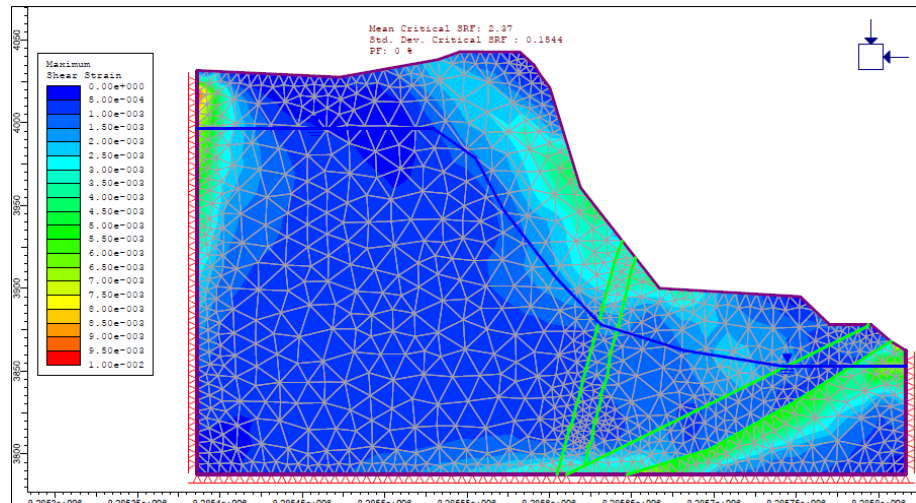

Imagen 4: Análisis de estabilidad probabilístico por el método de elementos finitos en condiciones estáticas.

SRF en condiciones seudoestáticas fue 1.21, con una probabilidad de falla de $2.98 \%$. (Imagen 5 ).

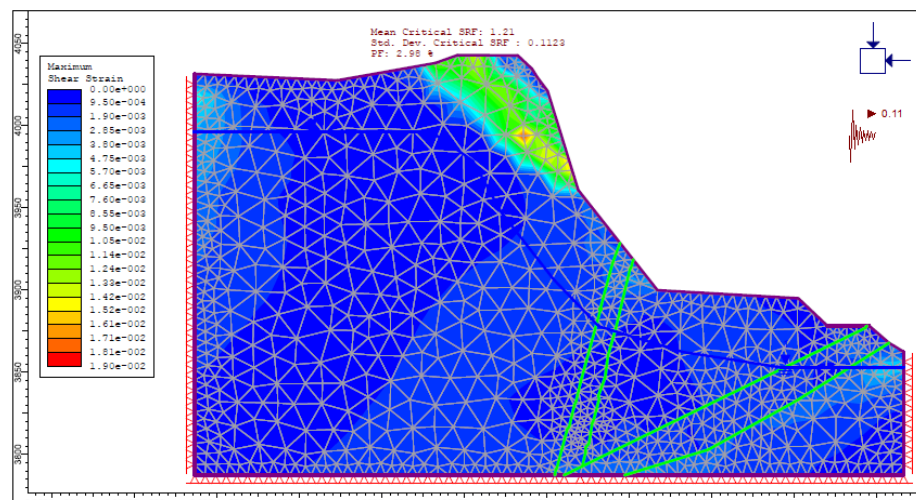

Imagen 5: Análisis de estabilidad probabilístico por el método de elementos finitos en condiciones seudoestáticas.

\section{Discusión}

Los resultados obtenidos son aceptables teniendo en cuenta la formación de grietas de tracción y el aparente modelo de ruptura por volteo directo en el campo.

\section{Conclusiones}

Los resultados obtenidos en el análisis cinemático nos muestran que tenemos la probabilidad máxima de falla por rotura de volteo directo igual a $44.41 \%$. Considerando este resultado confiable en la identificación del modo de falla en el talud y corroboramos este resultado con la presencia de grietas de tracción en la cresta del talud Espinal.

Los valores de seguridad promedio dado por los análisis probabilísticos por métodos numéricos en SRF son; para condiciones estáticas FS o SRF 2.27 y PF $0.0 \%$ y para condiciones seudoestáticas FS o SRF 1.21 y PF $2.98 \%$. Por lo cual concluimos que el talud se encuentra estable en condiciones estáticas y seudoestáticas.

La estabilidad del talud de la cantera Espinal dependerá de los factores desencadenantes tales como las fuertes precipitaciones y los movimientos sísmicos en la zona.

\section{Agradecimientos}

De manera general a las instituciones siguientes, Universidad Nacional del Altiplano, Escuela Profesional de Ingeniería de Minas, Escuela Profesional de Ingeniería Geológica y The Woman's Auxiliary to the American Institute of Mining, Metallurgical, and Petroleum Engineers (WAAIME).

De manera especial a las siguientes personas. Mario Cuentas, Erasmo Carnero, Gonzalo Carnero, Wilson Suaña, Luis Tejada, Arturo Chayña, Sandra Tica, Felipe Loaisa, Heydi Holguin, Lizet Llanos, Sayda Japura, Bernardino Valeriano, Ruth Nina, Elsa Mamani y a Luis Jordá por la revisión del artículo.

\section{Referencias}

[1] Bolaños, L.A. Monroy, C.O. 2004, Espectros de Peligro Sísmico Uniforme, PUCP, Tesis.

[2] Goodman, R.E. 1980. Introduction to Rock Mechanics, John Wiley, Toronto, Canada.

[3] Hudson, J.A. and Harrison, J.P. 1997. Engineering Rock Mechanics - An Introduction to the Principles, Pergamon Press.

[4] Hammah, R.E. and Yacoub, T.E. 2009. Probabilistic Slope Analysis with the Finite Element Method, In Proceedings the 43rd US Rock Mechanics Symposium and 4th U.S.Canada Rock Mechanics Symposium, Asheville.

[5] Read, J. y Stacey, P. 2009. Guidelines for open pit slope design, Editorial CSIRO PUBLISHING, Australia.

[6] Ramírez, O.P. y Alejano, M.L. 2004. Mecánica de Rocas: Fundamentos e Ingeniería de Taludes, Red DESIR, Madrid -España.

[7] Wyllie, C.D. and Mah, W.C. 2004. Rock Slope Engineering Civil and Mining, Spon Press Taylor \& Francis Group, New York. 